\title{
Two-Stage Fuzzy Logic Model for Cloud Service Supplier Selection and Evaluation
}

\author{
Marija Paunović $\mathbb{D},{ }^{1}$ Nebojša M. Ralević $\mathbb{D}^{1},{ }^{1}$ Vladimir Gajovićc $\mathbb{D}{ }^{2}$ \\ Biljana Mladenović Vojinović $\mathbb{D}^{1},{ }^{1}$ and Olivera Milutinović ${ }^{3}{ }^{3}$ \\ ${ }^{1}$ Department of Fundamentals Sciences, Faculty of Technical Sciences, University of Novi Sad, 21000 Novi Sad, Serbia \\ ${ }^{2}$ Dunav Insurance Company, 11000 Belgrade, Serbia \\ ${ }^{3}$ Megatrend University, 11000 Belgrade, Serbia
}

Correspondence should be addressed to Marija Paunović; majap@rcub.bg.ac.rs

Received 17 November 2017; Revised 8 June 2018; Accepted 12 June 2018; Published 9 July 2018

Academic Editor: Thomas Hanne

Copyright (C) 2018 Marija Paunović et al. This is an open access article distributed under the Creative Commons Attribution License, which permits unrestricted use, distribution, and reproduction in any medium, provided the original work is properly cited.

Dynamicity of the cloud computing system, complexity of real-world decision-making problems, and nontransparent market imply numerous difficulties in finding the corresponding solution for cloud supplier evaluation and selection. Due to that reason, there is a need to develop a model that can contribute to increase of reliability of evaluation and reduction of subjectivity of the decisionmaker. Having in mind the aforementioned, the authors proposed a new concept to model a cloud supplier evaluation based on technology perspective. Here, we developed a model that combined fuzzy techniques with implemented simulation of model parameters. The proposed method for cloud supplier evaluation is flexible with an objective evaluation and ensures more precise numerical value. Numerical examples are presented to demonstrate the effectiveness and pragmatism of the proposed approach.

\section{Introduction}

Cloud technologies, which enable new methods for provision of services and products by innovative and technological possibilities, are widely used all around us. Cloud service is a collection of computing; it usually refers to a dynamic and scalable virtualized resource. The quality of cloud service supplier is an important factor affecting the development of cloud computing. Due to increasing userspecific requirements for cloud services and differentiated quality of cloud services from numerous cloud service suppliers, selection of an appropriate cloud service supplier, based on technology, becomes a significant challenge for decision-makers.

Cloud service supplier uses different techniques such as ontologies, languages, standards, and tree structure and there is not a unified service description that describes all cloud service types, IaaS, PaaS, and SaaS. Apart from that, dynamicity of cloud computing and constant adding of new services and applications additionally make selection of the best cloud service supplier harder under the background of cloud computing emergence. Another problem in evaluation and service supplier selection is selection of a flexible and reliable model that enables fast implementation of new information.

Various mathematical techniques have been used by the researchers for selection and evaluation of suppliers. There are many evaluation methods in domain of cloud security engineering and cloud services security $[1,2]$, the quality of service [3-5], cloud storage [6], cloud manufacturing [7], and so on. Multicriteria decision-making (MCDM) allows decision-makers to face complex decision-making situations involving multiple, usually conflicting decision criteria, which include quantitative and/or qualitative aspects in a decision-making process. AHP method takes a significant place during selection of MCDM techniques for supplier evaluation and selection [8]. Critics of this method indicate that significance of elements presents only some sort of arithmetic accuracy that does not reflect real or objective evaluation. Also, when judgment matrix is not consistent, the adjustment is often with blindness and extensive calculation. Authors often combine this and other methods in order to 

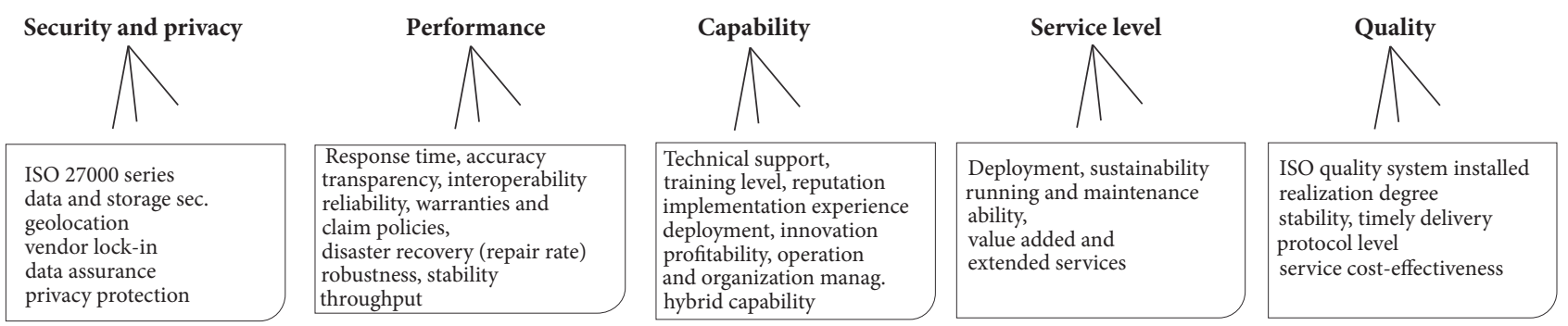

FIGURE 1: Cloud supplier selection and evaluation criteria and subcriteria (indexes).

overcome the stated deficiencies [9-12] and more can be seen in review paper of Le Sun [13].

In realistic environment of supplier selection and evaluation, a decision-maker must take into account different uncertain factors. Fuzzy-logic-based approach and decisionmaking problems under linguistic environment have been widely used in all areas of science and are one of the tools used for supplier selection $[14,15]$. Authors are trying to resolve the complexity of supplier selection problem by applying hybrid (integrated) models. The research of Simić [16] presents how fuzzy set theory, fuzzy decision-making, and hybrid solutions based on fuzzy mathematics can be used in the various models for supplier assessment and selection in a 50-year period. Comprehensive paper has shown that fuzzy hybrid approaches can be used to solve very complex real-world decision-making problems such as supplier assessment, ranking, and supplier selection. Min and Due [17] used a Group Decision-Making Model Based on Regression Method with Hesitant Fuzzy Preference Relations for supplier selection problem. Pang et al. [18] proposed A Fuzzy-Grey Multicriteria Decision-Making Approach for Green Supplier Selection. A review conducted by Mardani et al. [19] on fuzzy multiple criteria decision-making techniques and applications showed that the technique of Fuzzy Analytic Hierarchy Process (FAHP) was the most used one among MCDA techniques that employed fuzzy decision-making tools and approaches. Zhi Li et al. proposed mathematical model for supplier selection in the purchasing stage [20]. Their SSOA model comprises two processes, Fuzzy Extent Analytic Hierarchy Process (FEAHP) with multiobjective dynamic linear programming technique. Cloud service supplier selection problem under the background of cloud computing emergence, an integrated group of decision methods, based on SVM, TFN-RS-AHP, and TOPSIS-CD, was suggested by Liui et al. [21].

The motivation for the present work is the recognized absence and need for flexible but reliable model for cloud service supplier evaluation and selection. We provide two-stage fuzzy logic (2sFL) model, based on FAHP and fuzzy logic approaches for cloud supplier evaluation from technology perspective. The model enables a relatively simple correction of model parameters. This approach helps decision-makers in extracting and keeping expert knowledge in the system with opportunity to choose different operators, which leads to flexibility in the decision-making process. One more specificity of methods lies in implemented simulation of model parameters in order to ensure more precise numerical value. The proposed method is based on a new model that can take into account many factors in either part of the observed system or the whole, as well as their mutual relations on the goal of evaluation.

\section{Model Description}

The basic idea in developing the model was to evaluate, in a quality manner, cloud service supplier so as to take into account different decision elements (variables), their mutual relationship, relative importance, and impact on the whole. The model comprises two stages.

The first stage refers to establishment of input parameters and a hierarchical structure of the problem. FAHP method includes identification of goals and criteria that are compared and evaluated, evaluation based on comparison of pairs of elements on each hierarchy level, and synthesis of results based on comparison of criteria on all hierarchy levels. According to this method, the objective is defined on top of the hierarchical structure of the observed problem. Criteria that can be further broken down into subcriteria, that is, new hierarchy levels, are defined on the next level. Cloud computing is characterized by various factors and was examined from different perspectives (technological, business issues, applications, and general aspects) [22]. The SMI framework [23] contains 7 factors and 51 subfactors, and it is not expected that the decision-makers will want or need to use measures for all of the attributes. It is common to select factors and subfactors with greatest importance in decision process, which are the most relevant to their research. Lian-hui Li [21] proposed hierarchical framework with 4 characteristics from the technology perspective, and for the purpose of this work we took into account a criterion referring to security and privacy technology.

Five major aspects of cloud service supplier from the technology perspective are performance, capability, service level, quality and security, and privacy (see Figure 1). The complexity of business systems and processes is mainly dependent on the features of their subsystems and relations between subsystems. The quantity and the character of these relations inside the system are reflected on its structure and the number of operations that should be enabled by cloud service. The availability of information and experience generated from the previous and often limited time period influences the final decision of choice. For this reason, IT, engineering and technical knowledge, intuition, and the expert experience are often used in practice. Also, some 


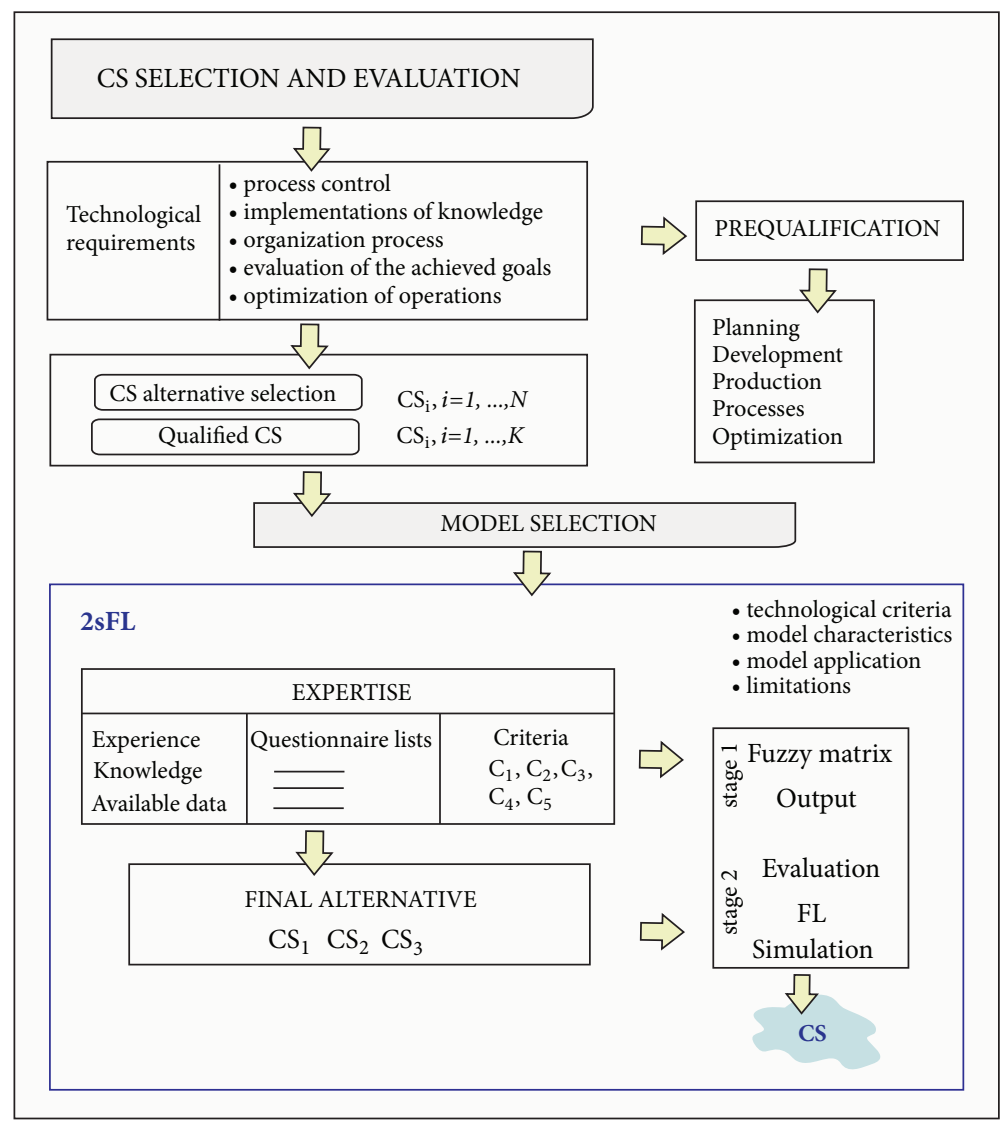

FIgURE 2: The selection and evaluation model.

unknown and rare situations and problems may arise in practice, which can neither be foreseen nor prevented but can have impact on the work of business systems. Due to these reasons, the proposed model, which directly accepts the expert knowledge through TFN score matrices and enables minimisation of subjectivity of expert assessments through fuzzy logic and simulation of variables, is better solution in some cases compared to others, especially in the situation when it is not possible to identify or solve all uncertainties (Figure 2). This method is used in cases of high complexities and existence of numerous impact parameters, when special features of decision problems exist and so forth.

When choosing criteria and subcriteria, what should be kept in mind is the complexity of cloud services and the fact that in many cases it assumes underlying cloud supply chain (existence of underlying cloud providers). If we observe security controls, they cannot be always implemented in the same way and as expected. Also, conventional/expected security controls could be compensated with alternative security controls. This causes that whole assessment process cannot be completely performed objectively, but that subjective impression of cloud assessor will always be included.

The complexity also depends on the delivery and deployment cloud model. For example, if the company has a very low risk appetite, it will not accept deployment models other than private cloud deployment models.
Considering the fact that data about elements are often not available or sufficiently reliable, for assessment of the value of model inputs experience, intuition and expert knowledge about the elements and their impact and classification on the observed process are used. Input values are expert estimates of relationship between values expressed by fuzzy numbers and output is significance of each element and process in whole. Application of fuzzy system leads to the improvement of the value supplier services. The output is the total service assessment for every specific criterion, according to the defined value scale, based on significance of every element.

Model is carried out in two phases, and each phase consists of several steps as follows.

Stage 1. Input values are expert estimates of relationship between values expressed by fuzzy numbers and output is significance of each element and total evaluation. Today we have several formulations of the fuzzy analytical method suggested by various authors. In this model, we use Chang's method, which can be described through the following steps $[24,25]$.

Step 1. The expert determines the value $m_{i j}$, for elements $i$ and $j$, where $m_{i j}$ is a triangular fuzzy number with parameters $a_{i j}, b_{i j}$, and $c_{i j}$. They are least possible values, respectively, and a TFN is represented as $\left(a_{i j}, b_{i j}, c_{i j}\right)$. 
Step 2. Summarize rows of the matrix $M=\left(m_{i j}\right)_{n \times n}$ so as to obtain values

$$
R S_{i}=\sum_{j=1}^{n} M_{i j}=\left(\sum_{j=1}^{n} a_{i j}, \sum_{j=1}^{n} b_{i j}, \sum_{j=1}^{n} c_{i j}\right)
$$

Normalize value $R S_{i}$ according to the equation

$$
\begin{aligned}
& S_{i}= \frac{R S_{i}}{\sum_{j=1}^{n} R S_{j}} \\
&=\left(\frac{\sum_{j=1}^{n} a_{i j}}{\sum_{k=1}^{n} \sum_{j=1}^{n} c_{k j}}, \frac{\sum_{j=1}^{n} b_{i j}}{\sum_{k=1}^{n} \sum_{j=1}^{n} b_{k j}}, \frac{\sum_{j=1}^{n} c_{i j}}{\sum_{k=1}^{n} \sum_{j=1}^{n} a_{k j}}\right), \\
& i=1, \ldots, n
\end{aligned}
$$

Step 3. The degree of probability that $S_{i} \geq S_{j}$ compared to relation $S_{i} \geq S_{j}$, where $S_{i}=\left(a_{i}, b_{i}, c_{i}\right)$ and $S_{j}=\left(a_{j}, b_{j}, c_{j}\right)$, is

$$
\begin{aligned}
& V\left(S_{i} \geq S_{j}\right) \\
& = \begin{cases}1, & \text { if } b_{i} \geq b_{j} \\
\frac{c_{i}-a_{j}}{\left(c_{i}-b_{i}\right)+\left(b_{j}-a_{j}\right)}, & \text { if } a_{j} \leq c_{i}, i, j=1, \ldots, n ; j \neq i \\
0, & \text { other }\end{cases}
\end{aligned}
$$

Determine the probability that the fuzzy number $S_{i}$ is greater than other fuzzy numbers according to the equation:

$$
\begin{aligned}
V & \left(S_{i} \geq S_{j} \mid j=1, \ldots, n ; j \neq i\right) \\
& =\min _{j \in\{1, \ldots, n\}, j \neq i} V\left(S_{i} \geq S_{j}\right), \quad i=1, \ldots, n
\end{aligned}
$$

Step 4. Determine priority vectors $W=\left(w_{1}, \ldots, w_{n}\right)^{T}$ of comparison matrix of the fuzzy value $M$ as

$$
\begin{aligned}
& w_{i}=\frac{V\left(S_{i} \geq S_{j} \mid j=1, \ldots, n ; j \neq i\right)}{\sum_{k=1}^{n} V\left(S_{k} \geq S_{j} \mid j=1, \ldots, n ; j \neq k\right)}, \\
& i=1, \ldots, n
\end{aligned}
$$

Output result of this model is the value of priority, significance $S_{i}$ of each element and the total value $\left(W=w_{1}\right.$, low; $w_{2}$, medium; $\mathrm{w}_{3}$, high).

Stage 2 (application of fuzzy logic and approximate reasoning algorithms). In order to define an approximate reasoning algorithm, it is necessary to observe variables that are present in premises and consequences of certain rules and describe them by adequate fuzzy sets. Fuzzy system for assessment includes the following steps.

Step 1 (defining input variables and output variables). Fuzzy system enables supplier assessment based on qualification of all elements. Interval of value $I_{i}$ for each element and each level $i$ is determined based on the value of the priority of elements obtained in Stage 1.

Step 2 (defining the membership functions for selected variables). Each element can be described by fuzzy sets as low, medium, and high. The shape of membership functions of fuzzy sets $X_{L}, X_{M}$, and $X_{H}$ is the same for all elements. Values $\mathrm{x}_{1}, \mathrm{x}_{2}, \mathrm{x}_{3}, \mathrm{x}_{4}, \mathrm{x}_{5}$, and $\mathrm{x}_{6}$ for left and right border and the value with the highest level of membership of fuzzy numbers are different for each element.

Membership functions are

$$
\begin{gathered}
\mu_{L}(x)= \begin{cases}\frac{x_{2}-x}{x_{2}}, & 0 \leq x \leq x_{2} \\
0, & x>x_{2}\end{cases} \\
\mu_{X_{M}}(x)= \begin{cases}0, & x \leq x_{1} \\
\frac{x-x_{1}}{x_{3}-x_{1}}, & x_{1}<x \leq x_{3} \\
\frac{x_{5}-x}{x_{5}-x_{3}}, & x_{3}<x \leq x_{5} \\
0, & x>x_{5}\end{cases} \\
\mu_{H}(x)= \begin{cases}0, & x \leq x_{4} \\
\frac{x-x_{4}}{x_{6}-x_{4}}, & x_{4}<x \leq x_{6} \\
0, & x>x_{6}\end{cases}
\end{gathered}
$$

The output value of the fuzzy system is the total assessment in specific process (TA) and membership functions of fuzzy sets: $\mathrm{Y}_{\mathrm{VL}}$, very low; $\mathrm{YL}$, low; $\mathrm{Y}_{\mathrm{M}}$, medium; $\mathrm{Y}_{\mathrm{H}}$, high; and $\mathrm{Y}_{\mathrm{VH}}$, very high. Membership functions are defined in the range $[0,100]$ by the following relations:

$$
\mu_{Y_{V L}}(y)= \begin{cases}\frac{20-y}{20}, & 0 \leq y \leq 20 \\ 0, & y>20\end{cases}
$$$$
\mu_{Y_{L}}(y)= \begin{cases}0, & y \leq 5 \\ \frac{y-5}{20}, & 5<y \leq 25 \\ \frac{45-y}{20}, & 25<y \leq 45 \\ 0, & y>45\end{cases}
$$$$
\mu_{Y_{M}}(y)= \begin{cases}0, & y \leq 30 \\ \frac{y-30}{20}, & 30<y \leq 50 \\ \frac{70-y}{20}, & 50<y \leq 70 \\ 0, & y>70\end{cases}
$$$$
\mu_{Y_{H}}(y)= \begin{cases}0, & y \leq 55 \\ \frac{y-55}{20}, & 55<y \leq 75 \\ \frac{95-y}{20}, & 75<y \leq 95 \\ 0, & y>95\end{cases}
$$ 


$$
\mu_{Y_{V H}}(y)= \begin{cases}0, & y \leq 80 \\ \frac{y-80}{20}, & 80<y \leq 100\end{cases}
$$

Step 3 (determining intervals of values of input and output variable). Based on defined interval of value $I_{i}$ of input variables, left and right boundaries can be set, and the values with the highest level of membership of fuzzy numbers $\mathrm{X}_{\mathrm{L}}$, $\mathrm{X}_{\mathrm{M}}$, and $\mathrm{X}_{\mathrm{H}}$, for each element, are as follows: $\mathrm{X}_{\mathrm{i} 1}=0.1 \mathrm{I}_{\mathrm{i}}, \mathrm{X}_{\mathrm{i} 2}$ $=0.3 \mathrm{I}_{\mathrm{i}}, \mathrm{x}_{\mathrm{i} 3}=0.5 \mathrm{I}_{\mathrm{i}}, \mathrm{x}_{\mathrm{i} 4}=0.7 \mathrm{I}_{\mathrm{i}}, \mathrm{x}_{\mathrm{i} 5}=0.9 \mathrm{I}_{\mathrm{i}}$, and $\mathrm{x}_{\mathrm{i} 6}=\mathrm{I}_{\mathrm{i}}$. The output of the fuzzy system can be in the interval $(0,100)$ and represents the value of the total assessments.

The model was tested on a large number of hypothetical examples. Input parameters were obtained by generating random numbers for each element in accordance with output data in Stage 1. Each element is simulated as an independent variable with uniform distribution within interval $\left(0, \mathrm{I}_{\mathrm{i}}\right), X \sim$ $R\left(0, I_{i}\right)$. For level $\mathrm{i}$, supplier $\mathrm{j}$, and each element $\mathrm{x}_{\mathrm{ij}}$, the process would be as follows:

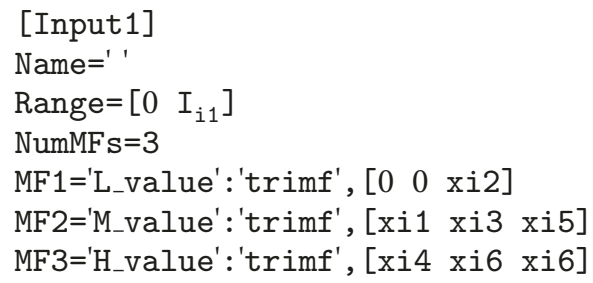

Step 4 (define the approximate reasoning algorithm). Let $V=$ $V_{1} \times V_{2} \times \ldots \times V_{n} \subset R_{n}$ be the input space and let $T \subset R$ be the output space. Rules are defined to show the relations between all possible combinations of input and output variables in a reasonable, realistic, and efficient manner. These rules define a mapping from fuzzy sets in the input universe of discourse $V \subset R_{\mathrm{n}}$ to fuzzy sets in the output universe of discourse $T \subset$ $R$, based on fuzzy logic principles. The fuzzy if-then rules are defined as follows:

$$
\begin{gathered}
\mathrm{R}^{(1)}: \text { IF } \mathrm{v}_{1} \text { is } \mathrm{L}_{1}^{l} \text { and IF } \mathrm{v}_{2} \text { is } \mathrm{L}_{2}^{l} \text { and } \\
\cdots \text { IF } \mathrm{v}_{\mathrm{n}} \text { is } \mathrm{L}_{n}^{l} \text { THEN } \mathrm{t} \text { is } \mathrm{T}^{l}
\end{gathered}
$$

where $L_{i}^{l} \mathrm{i}=1,2, \ldots, \mathrm{n}$, and $\mathrm{T}^{l}$ are fuzzy sets in $V n \subset R n$, respectively, and $v=\left(v_{1}, v_{2}, . . v_{n}\right)^{T} \in V$ and $t \in T$ are input and output linguistic variables that belong to the input and output universes, respectively. Let $\mathrm{M}$ be the number of rules in the fuzzy rule base $(1=1,2, \ldots, M)$ and each fuzzy rule represents fuzzy set $L_{1}^{l}, L_{2}^{l}, \ldots, L_{n}^{l} \longrightarrow T^{l}$ for $1=1,2, \ldots, \mathrm{M}$, in the product space $\mathrm{V} \times \mathrm{T}$; then $\mathrm{M}$ represents a set of variations with repetition.

Approximate reasoning includes the process of aggregation based on which the output values of all fuzzy rules compress into a single fuzzy set. In this fuzzy system, authors used the Mamdani technique.

The t-norm (minimum) is usually adopted for the logic connective "and," as expressed by the following equation:

$$
\mu_{A}(x) \text { and } \mu_{B}(x)=\min \left\{\mu_{A}(x), \mu_{B}(x)\right\}
$$

For the logic connective "or" s-norm (maximum) is usually represented by

$$
\mu_{A}(x) \text { or } \mu_{B}(x)=\max \left\{\mu_{A}(x), \mu_{B}(x)\right\}
$$

For each activated rule, the inference machine applies an implication relation $\mathrm{R}$ between the fuzzy number resulting from the logic operations and the consequent. Implication operator, commonly in use, is the minimum (Mamdani), expressed in the following equation:

$$
\mu_{R}(x, y)=\min \left\{\mu_{A}(x), \mu_{B}(x)\right\}
$$

Alternative operators are Zadeh Max-Min and Larsen multiplication.

The output fuzzy number of each rule is explained by the composition between a fuzzy singleton and the implication relation. Commonly used composition operators of fuzzy relations are Max-Min, Max-prod, and Max-Media in the following equations [26]:

$$
\begin{gathered}
\operatorname{SoR}(x, y)=\max \left\{\min \left(\mu_{s}(x, y), \mu_{R}(y, z)\right)\right\} \\
\operatorname{S.R}(x, z)=\max \left\{\left(\mu_{s}(x, y) * \mu_{R}(y, z)\right)\right\} \\
\operatorname{S} \oplus \mathrm{R}(x, z)=\max \left\{\frac{1}{2}\left(\mu_{s}(x, y)+\mu_{R}(y, z)\right)\right\}
\end{gathered}
$$

Step 5 (defuzzification). In this model, we applied the centroid method.

$$
Z_{\mathrm{COA}}=\frac{\int_{z} \mu_{A}(Z) Z d z}{\int_{z} \mu_{A}(Z) d z}
$$

\section{Implementation of the Model with Numerical Example}

The packaging company conducts business in $100+$ countries. It uses numerous systems to execute functions of the company, including numerous connections to customers, suppliers, and other partners. The company employed a relatively streamlined, mainly qualitative methodology to assess supplier. The methodology followed defined steps for analysing supplier and involved a number of standardized, systematic tools and procedures to compile and analyse data and generate reports.

Prequalification and the selection of the cloud suppliers are done first within the business stakeholders of the company 
in accordance with their business needs. Qualified suppliers are then considered from the technological point of view by experts and engineers in the technical sector, considering in particular the security controls implemented into the service offered by cloud suppliers in relation to all five criteria. The selection of cloud service supplier is made in relation to the assignment of implemented controls, whether they correspond to the company's criteria and their defined policies or procedures related to the data classification and handling. Assessment is mainly performed based on questionnaire lists that will be fulfilled by cloud suppliers and supporting documentation.

The expert team and engineers used the cloud technology on comprehensive and operable principles and, to their best knowledge and experience, selected 3 suppliers from K collaborative suppliers for innovation architecture engineering solution and created the judgment matrices.

Stage 1. Experts determine pairwise comparison of the different criterion relevant to the overall objective using triangular fuzzy numbers. The value of fuzzy extent with respect to each criterion is calculated by using Step 2 from Stage 1 and the formula for algebraic operations of the fuzzy set. Matrix of the elements comparison and TFN score matrices of 3 suppliers given by experts are $C S_{k}=\left(m_{i j}\right)_{5 \times 5}, k=1,2,3$, with $m_{i j}=$ $\left(a_{i j}, b_{i j}, c_{i j}\right)$.

$$
C S_{1}=\left[\begin{array}{rrrrr}
(1,1,1) & (1,3,5) & (1,3,5) & (3,5,7) & (3,5,7) \\
& (1,1,1) & (1,3,5) & (1,3,5) & (1,3,5) \\
& & (1,1,1) & (3,5,7) & (1,1,1) \\
& & & (1,1,1) & (1,1,3) \\
& & & & (1,1,1)
\end{array}\right]
$$

$$
\begin{aligned}
& C S_{2} \\
& =\left[\begin{array}{lllll}
(1,1,1) & (3,5,7) & (0.33,1,1) & (3,5,7) & (3,5,7) \\
& (1,1,1) & (1,3,5) & (1,3,5) & (1,3,5) \\
& & (1,1,1) & (1,3,5) & (1,1,3) \\
& & & (1,1,1) & (1,1,3) \\
& & & & (1,1,1)
\end{array}\right]
\end{aligned}
$$

$$
\begin{aligned}
& C S_{3} \\
& =\left[\begin{array}{llllc}
(1,1,1) & (1,1,3) & (2,3,4) & (3,4,5) & (2,3,4) \\
& (1,1,1) & (2,3,4) & (3,4,5) & (3,4,5) \\
& & (1,1,1) & (2,3,4) & (0.2,0.33,1) \\
& & & (1,1,1) & (1,1,3) \\
& & & & (1,1,1)
\end{array}\right]
\end{aligned}
$$

The consistency of the pairwise judgment of each comparison matrix is also checked using the calculation method of consistency index. The degree of probability can be determined by Step 3 of Stage 1 .

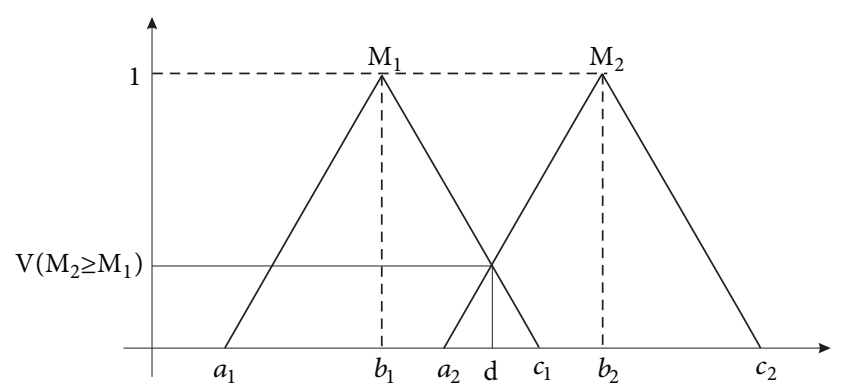

Figure 3: The intersection between $M_{1}$ and $M_{2}$.

We obtain the following values for CS1:

$$
\begin{aligned}
& V\left(S_{1} \geq S_{1}\right)=1, \\
& V\left(S_{1} \geq S_{2}\right)=1.196, \\
& V\left(S_{1} \geq S_{3}\right)=1.306, \\
& V\left(S_{1} \geq S_{4}\right)=1.518, \\
& V\left(S_{1} \geq S_{5}\right)=1.478, \\
& V\left(S_{2} \geq S_{1}\right)=0.780, \\
& V\left(S_{2} \geq S_{2}\right)=1, \\
& V\left(S_{2} \geq S_{3}\right)=1.114, \\
& V\left(S_{2} \geq S_{4}\right)=1.374, \\
& V\left(S_{2} \geq S_{5}\right)=1.324,
\end{aligned}
$$

and continuing the process,

$$
\begin{aligned}
& V\left(S_{5} \geq S_{1}\right)=0.108, \\
& V\left(S_{5} \geq S_{2}\right)=0.413, \\
& V\left(S_{5} \geq S_{3}\right)=0.491, \\
& V\left(S_{5} \geq S_{4}\right)=1.157, \\
& V\left(S_{5} \geq S_{5}\right)=1 .
\end{aligned}
$$

For illustration, Figure 3 presents the intersection between $\mathrm{M}_{1}$ and $\mathrm{M}_{2}$.

Minimum degree of possibility can be stated as $d^{\prime}\left(S_{1}\right)=$ $\min V\left(S_{1} \geq S_{2}, S_{3}, S_{4}, S_{5}\right)=1$, and similarly $d^{\prime}\left(S_{2}\right)=0.780$, $d^{\prime}\left(S_{3}\right)=0.586, d^{\prime}\left(S_{4}\right)=0.217$, and $d^{\prime}\left(S_{5}\right)=0.108$.

The same process is applied to all suppliers and results are shown in Table 1.

Table 2 shows calculated values of the resulting vector of priorities $\mathrm{W}$ for each supplier.

Output result of this model is the value of priority, significance $S_{i}$ of each element and the total value of security $\mathrm{W}$ $=\mathrm{w}_{1}$, low; $\mathrm{w}_{2}$, medium; $\mathrm{w}_{3}$, high. Afterwards, experts define elements of comparison matrix for each level compared to each element. By applying relations from Stage 1, we calculate 
TABLE 1: The degree of probability for $\mathrm{CS}_{1}, \mathrm{CS}_{2}$, and $\mathrm{CS}_{3}$.

\begin{tabular}{|c|c|c|c|}
\hline & $\mathrm{CS}_{1}$ & $\mathrm{CS}_{2}$ & $\mathrm{CS}_{3}$ \\
\hline sls1 & 1 & 1 & 1 \\
\hline s1s2 & 1.196084 & 1.225806 & 0.944482 \\
\hline s1s3 & 1.305547 & 1.406946 & 1.496616 \\
\hline sls4 & 1.518374 & 1.593957 & 1.722365 \\
\hline s1s5 & 1.477589 & 1.543012 & 1.41821 \\
\hline s2s1 & 0.779941 & 0.759967 & 1.067317 \\
\hline $\mathrm{s} 2 \mathrm{~s} 2$ & 1 & 1 & 1 \\
\hline $\mathrm{s} 2 \mathrm{~s} 3$ & 1.114353 & 1.179423 & 1.683952 \\
\hline $\mathrm{s} 2 \mathrm{~s} 4$ & 1.374472 & 1.383257 & 1.987905 \\
\hline s2s5 & 1.324265 & 1.331033 & 1.572959 \\
\hline s3s1 & 0.585949 & 0.596962 & 0.318209 \\
\hline $\mathrm{s} 3 \mathrm{~s} 2$ & 0.859789 & 0.836086 & 0.274406 \\
\hline $\mathrm{s} 3 \mathrm{~s} 3$ & 1 & 1 & 1 \\
\hline s3s 4 & 1.393349 & 1.200729 & 1.379907 \\
\hline s3s5 & 1.312726 & 1.153214 & 0.930194 \\
\hline s4s1 & 0.216659 & 0.234851 & 0.071214 \\
\hline $\mathrm{s} 4 \mathrm{~s} 2$ & 0.482643 & 0.529772 & 0.043399 \\
\hline $\mathrm{s} 4 \mathrm{~s} 3$ & 0.560987 & 0.703511 & 0.677156 \\
\hline $\mathrm{s} 4 \mathrm{~s} 4$ & 1 & 1 & 1 \\
\hline s4s5 & 0.909993 & 0.935154 & 0.654477 \\
\hline s5s1 & 0.107991 & 0.062627 & 0.408564 \\
\hline $\mathrm{s} 5 \mathrm{~s} 2$ & 0.4125 & 0.419801 & 0.361055 \\
\hline $\mathrm{s} 5 \mathrm{~s} 3$ & 0.49108 & 0.630968 & 1.075847 \\
\hline s5s 4 & 1.156943 & 1.12892 & 1.437524 \\
\hline s5s5 & 1 & 1 & 1 \\
\hline
\end{tabular}

TABLE 2: Resulting vector of priorities W.

\begin{tabular}{lccc}
\hline & CS1 & CS2 & CS3 \\
\hline Service security and privacy, v1 & 0.3717 & 0.3767 & 0.3600 \\
Supplier service level, v2 & 0.2899 & 0.2863 & 0.3812 \\
Supplier service quality, v3 & 0.2178 & 0.2249 & 0.1046 \\
Supplier capability, v4 & 0.0805 & 0.0885 & 0.0165 \\
Cloud service performance, v5 & 0.0401 & 0.0236 & 0.1376 \\
\hline
\end{tabular}

a local priority $w$, for each hierarchy level. Values of all matrix elements are expressed by triangular fuzzy numbers.

Stage 2. The parameters of membership functions for all input variables of fuzzy system and different supplier are shown in Table 3. The rules allow quantitative, qualitative, and judgmental data to be integrated in a uniform manner. Approximate reasoning algorithm includes 243 fuzzy rules that represent a set of variations with repetition of set $v_{1} \times v_{2} \times$ $\mathrm{v}_{3} \times \mathrm{v}_{4} \times \mathrm{v}_{5} \times \mathrm{TE}$ and levels (very low, low, medium, high, and very high).

The rules are defined as follows [27]:

$\mathrm{R}^{(1)}:$ IF $\mathrm{v}_{1}$ Low and $\mathrm{v}_{2}$ Low and $\mathrm{v}_{3}$ Low and $\mathrm{v}_{4}$ Low and $v_{5}$ Low THEN TE Very Low

$\mathrm{R}^{(2)}$ : IF $\mathrm{v}_{1}$ Low and $\mathrm{v}_{2}$ LOW and $\mathrm{v}_{3}$ Low and $\mathrm{v}_{4}$ and $\mathrm{v}_{5}$ Medium THEN TE Very Low
$\mathrm{R}^{(120)}$ : IF $\mathrm{v}_{1}$ Medium and $\mathrm{v}_{2}$ Medium and $\mathrm{v}_{3}$ Medium and $v_{4}$ Low and $v_{5}$ High THEN TE Medium

$\mathrm{R}^{(121)}$ : IF $\mathrm{v}_{1}$ Medium and $\mathrm{v}_{2}$ Medium and $\mathrm{v}_{3}$ Medium and $\mathrm{v}_{4}$ Medium and $\mathrm{v}_{5}$ Low THEN TE Low

...

$\mathrm{R}^{(242)}$ : IF $\mathrm{v}_{1}$ High and $\mathrm{v}_{2}$ High and $\mathrm{v}_{3}$ High and $\mathrm{v}_{4}$ High and $\mathrm{v}_{5}$ Medium THEN TE Very high

$\mathrm{R}^{(243)}$ : IF $\mathrm{v}_{1}$ High and $\mathrm{v}_{2}$ High and $\mathrm{v}_{3}$ High and $\mathrm{v}_{4}$ High and $v_{5}$ High THEN TE Very High

Simulation process is implemented in model and problem was solved by using MATLAB $^{\circledR}$ software. Each element is simulated as an independent variable with uniform distribution within interval $\left(0, I_{i}\right)$. For example, for variable $\mathrm{v}_{11}$ simulated on $X \sim R(0,0.3717)$ by generating random 


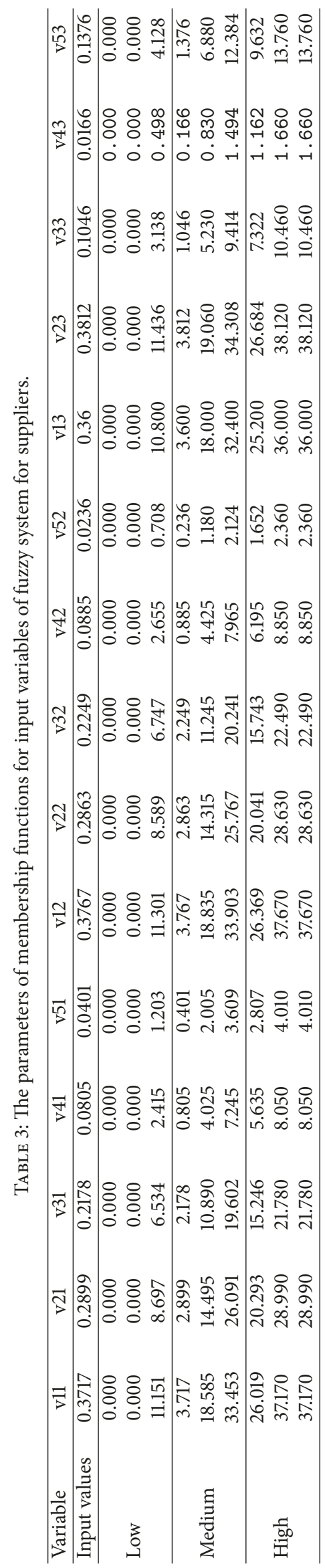




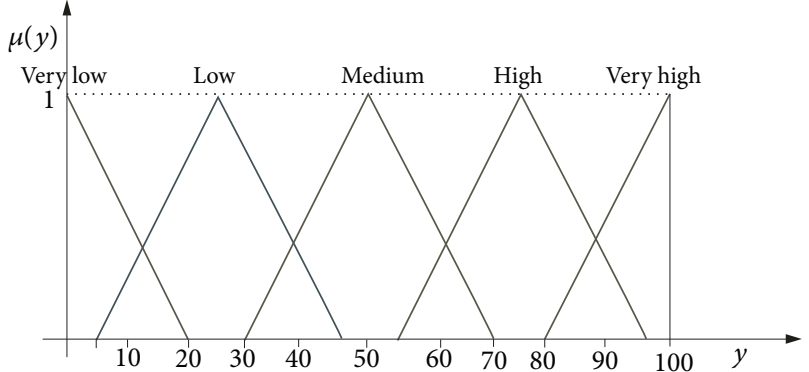

(a)

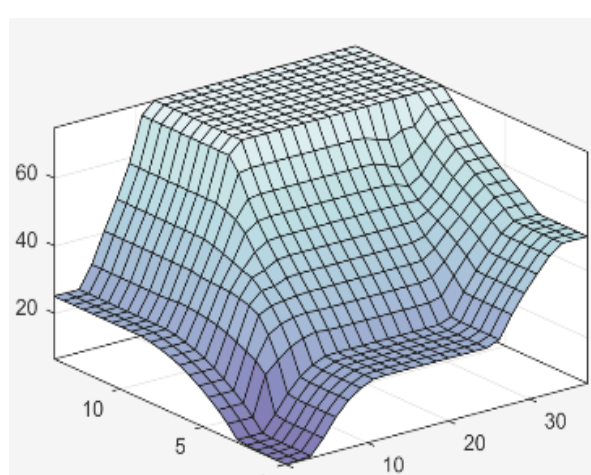

(b)

FIgURE 4: Value of the fuzzy system for $\mathrm{CS}_{1}$. (a) The output value in Stage 1. (b) Total evaluation of $\mathrm{CS}_{1}$.

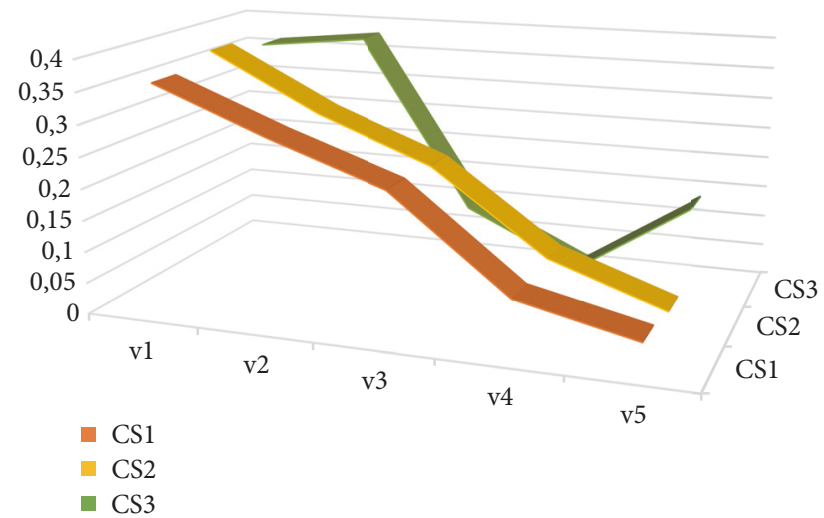

FIGURE 5: Cloud service supplier's comparative evaluation result.

numbers, 10000 sets of input data are simulated in order to obtain output data of a fuzzy model, that is, weight elements. The output value for $\mathrm{CS}_{1}$ in Stage 1 is shown in Figure 4(a), and total evaluation of $\mathrm{CS}_{1}$ is presented in Figure 4(b).

The same process is repeated for selected subelements of the system, if experts deem it necessary. Table 4 summarized the results obtained by model for each cloud service supplier in respect of five major aspects of cloud service supplier from the technology perspective. The highest value in service supplier evaluation process has security and quality for all 3 cloud service suppliers (see Figure 5). The first two suppliers have a rather similar evaluation of parameters, $v_{1}>v_{2}>$ $v_{3}>v_{4}>v_{5}$, while the third supplier has $v_{1}>v_{2}>v_{5}>$ $v_{3}>v_{4}$. According to its preferences, the company further recommends a supplier. In case of similar evaluation values, other factors can further be considered, such as financial ones.

The idea to minimize the subjectivity degree of decisionmakers is realized by using fuzzy logic model. Experts assess the value for each of total elements, while assessment of the total evaluation is realized by using a fuzzy model. Assessment of particular elements can be implemented by using different scales, whose value ranges can be adjusted to the practice and mapped to the value interval of corresponding fuzzy numbers. This approach, as a consequence, increases the accuracy of the assessment of each particular evaluation that belongs to a particular class.

Comparable evaluating results of the first suppliers according to FAHP, FANP, and our methods are shown in Table 5. Results of all three methods are generally consistent. Disadvantages of the FAHP method (Wang et al. [29]) which result in inconsistency in output data in our model are overcome by introduction of fuzzy logic. Partial treatment of various elements, analysis, and assessment of individual elements, which affects the total evaluation, leads to a reduction of inadequate estimations in decision-making and reduces the possibility of error. Simulation technique is able to model the dynamic behaviour of the cloud suppler and agile strategies with generating more precise numerical value. Proposed model is flexible and enables a relatively simple correction of input parameters and fast generation of values in specific system or processes.

\section{Conclusion}

Fast growing and nontransparent cloud computing market with various providers and specific models of services greatly makes their evaluation difficult in order to perform comparisons. Another problem is the interoperability between providers which is not achieved and often makes selection of a provider an irreversible process or its selection requires more efforts. We proposed two-stage fuzzy logic model, based on Fuzzy Analytic Hierarchy Process (FAHP) and fuzzy logic approaches for cloud supplier evaluation. In this paper, five major aspects of cloud service supplier from the technology perspective were applied to evaluate suppliers, while other critical factors could be added to this model. Defining fuzzy rules for the proposed model needs experience from field experts and experimental results to obtain effective results. Specificity of the method lies in implemented simulation of model parameters in order to ensure more precise numerical value. It should be mentioned that this study was limited to the cloud service supplier evaluation, but the model can be used in different areas of science and practice purpose. 
TABLE 4: Fuzzy system evaluation (simulation result).

\begin{tabular}{lccccc}
\hline Variable & $\mathrm{v}_{1}$ & $\mathrm{v}_{2}$ & $\mathrm{v}_{3}$ & $\mathrm{v}_{4}$ & 0.08255 \\
\hline $\mathrm{CS}_{1}$ & 0.36040 & 0.28975 & 0.22673 & 0.08506 & 0.02350 \\
$\mathrm{CS}_{2}$ & 0.38048 & 0.28885 & 0.21912 & 0.01610 & 0.13869 \\
$\mathrm{CS}_{3}$ & 0.36151 & 0.38121 & 0.10248 & $\mathrm{v}_{5}$ \\
\hline
\end{tabular}

TABLE 5: Comparison of results.

\begin{tabular}{lcccrr}
\hline Variable & $\mathrm{v}_{1}$ & $\mathrm{v}_{2}$ & $\mathrm{v}_{3}$ & $\mathrm{v}_{4}$ & \\
\hline FANP (Yu [28]) & 0.3544 & 0.2883 & 0.2104 & 0.1143 & 0.0326 \\
FAHP (Cnang [24]) & 0.3717 & 0.2899 & 0.2178 & 0.0805 & 0.0401 \\
Our model & 0.3604 & 0.2897 & 0.2267 & 0.0826 & 0.0406 \\
\hline
\end{tabular}

\section{Conflicts of Interest}

The authors declare that there are no conflicts of interest regarding the publication of this paper.

\section{Acknowledgments}

The second author acknowledges the financial support of the Ministry of Education, Science and Technological Development of the Republic of Serbia in the frame of projects applied under no. TR 34014 and no. ON 174009.

\section{References}

[1] S. A. Aljawarneh, A. Alawneh, and R. Jaradat, "Cloud security engineering: Early stages of SDLC," Future Generation Computer Systems, vol. 74, pp. 385-392, 2017.

[2] P. Junghanns, B. Fabian, and T. Ermakova, "Engineering of secure multi-cloud storage," Computers in Industry, vol. 83, pp. 108-120, 2016.

[3] M. Tang, X. Dai, J. Liu, and J. Chen, "Towards a trust evaluation middleware for cloud service selection," Future Generation Computer Systems, vol. 74, pp. 302-312, 2017.

[4] S. Ding, S. Yang, Y. Zhang, C. Liang, and C. Xia, "Combining QoS prediction and customer satisfaction estimation to solve cloud service trustworthiness evaluation problems," KnowledgeBased Systems, vol. 56, pp. 216-225, 2014.

[5] B. Kang, J. Wang, and D. Shao, "Attack on Privacy-Preserving Public Auditing Schemes for Cloud Storage," Mathematical Problems in Engineering, vol. 2017, Article ID 8062182, 2017.

[6] G. D. Gonçalves, I. Drago, A. B. Vieira, A. P. Couto da Silva, J. M. Almeida, and M. Mellia, "Workload models and performance evaluation of cloud storage services," Computer Networks, vol. 109, pp. 183-199, 2016.

[7] P. Wang, R. X. Gao, and Z. Fan, "Cloud Computing for Cloud Manufacturing: Benefits and Limitations," Journal of Manufacturing Science and Engineering, vol. 137, no. 4, Article ID 044002, 2015.

[8] S. H. Zyoud and D. Fuchs-Hanusch, "A bibliometric-based survey on AHP and TOPSIS techniques," Expert Systems with Applications, vol. 78, pp. 158-181, 2017.

[9] S. Ding, Z. Wang, D. Wu, and D. L. Olson, "Utilizing customer satisfaction in ranking prediction for personalized cloud service selection," Decision Support Systems, vol. 93, pp. 1-10, 2017.
[10] C. López and A. Ishizaka, "GAHPSort: A new group multicriteria decision method for sorting a large number of the cloud-based ERP solutions," Computers in Industry, vol. 92-93, pp. 12-24, 2017.

[11] Y. Wang, J. Wen, X. Wang, and W. Zhou, "Cloud service evaluation model based on trust and privacy-aware," Optik International Journal for Light and Electron Optics, vol. 134, pp. 269-279, 2017.

[12] A. Kokangul and Z. Susuz, "Integrated analytical hierarch process and mathematical programming to supplier selection problem with quantity discount," Applied Mathematical Modelling, vol. 33, no. 3, pp. 1417-1429, 2009.

[13] L. Sun, H. Dong, F. K. Hussain, O. K. Hussain, and E. Chang, "Cloud service selection: state-of-the-art and future research directions," Journal of Network and Computer Applications, vol. 45, pp. 134-150, 2014.

[14] B. Pardha Saradhi, N. Ravi Shankar, and C. Suryanarayana, "Novel distance measure in fuzzy TOPSIS for supply chain strategy based supplier selection," Mathematical Problems in Engineering, Article ID 7183407, p. 17, 2016.

[15] M. S. Amalnick, A. HakimiAsl, Y. A. Zanjirani, and M. HakimiAsl, "A Cloud Manufacturing Resource Allocation by Using an Integrated FAHP-FTOPSIS Approach in High-Tech Industries," Applied mathematics in engineering, management and technology, vol. 4, no. 1, pp. 1-8, 2016.

[16] D. Simić, I. Kovačević, V. Svirčević, and S. Simić, “50 years of fuzzy set theory and models for supplier assessment and selection: A literature review," Journal of Applied Logic, vol. 24, pp. 85-96, 2017.

[17] M. Xue and Y. Du, "A group decision-making model based on regression method with hesitant fuzzy preference relations," Mathematical Problems in Engineering, Article ID 6549791, p. 8, 2017.

[18] Q. Pang, T. Yang, M. Li, and Y. Shen, "A Fuzzy-Grey Multicriteria Decision Making Approach for Green Supplier Selection in Low-Carbon Supply Chain," Mathematical Problems in Engineering, Article ID 9653261, p. 9, 2017.

[19] A. Mardani, A. Jusoh, and E. K. Zavadskas, "Fuzzy multiple criteria decision-making techniques and applications - Two decades review from 1994 to 2014," Expert Systems with Applications, vol. 42, no. 8, pp. 4126-4148, 2015.

[20] Z. Li, W. K. Wong, and C. K. Kwong, "An integrated model of material supplier selection and order allocation using fuzzy extended AHP and multiobjective programming," Mathematical Problems in Engineering, vol. 2013, Article ID 363718, 14 pages, 2013. 
[21] L.-h. Li, J.-c. Hang, Y. Gao, and C.-y. Mu, "Using an Integrated Group Decision Method Based on SVM, TFN-RS-AHP, and TOPSIS-CD for Cloud Service Supplier Selection," Mathematical Problems in Engineering, Article ID 3143502, p. 14, 2017.

[22] H. Yang and M. Tate, "Where are we at with Cloud Computing? A Descriptive Literature Review," in Proceedings of the Australasian Conference on Information Systems, Melbourne, Australia, 2009.

[23] J. Siegel and J. Perdue, "Cloud services measures for global use: the Service Measurement Index (SMI)," in Proceedings of the Annual SRII Global Conference (SRII '12), pp. 411-415, San Jose, Calif, USA, July 2012.

[24] D. Y. Chang, "Applications of the extent analysis method on fuzzy AHP," European Journal of Operational Research, vol. 95, no. 3, pp. 649-655, 1996.

[25] G. Radivojević and V. Gajović, "Supply chain risk modeling by AHP and Fuzzy AHP methods," Journal of Risk Research, vol. 17, no. 3, pp. 337-352, 2014.

[26] W. Pedrycz and F. Gomide, Fuzzy systems engineering-toward human-centric computing, Wiley, New Jerse, USA, 2007.

[27] V. Gajović, M. Kerkez, and J. Kočović, "Modeling and simulation of logistic processes: risk assessment with a fuzzy logic technique," Simulation Transactions of the Society for Modeling and Simulation International, vol. 94, no. 6, pp. 507-518, 2017.

[28] R. Yu and G.-H. Tzeng, "A soft computing method for multicriteria decision making with dependence and feedback," Applied Mathematics and Computation, vol. 180, no. 1, pp. 63-75, 2006.

[29] Y. M. Wang, Y. Luo, and Z. Hua, "On the extent analysis method for fuzzy AHP and its applications," European Journal of Operational Research, vol. 186, no. 2, pp. 735-747, 2008. 


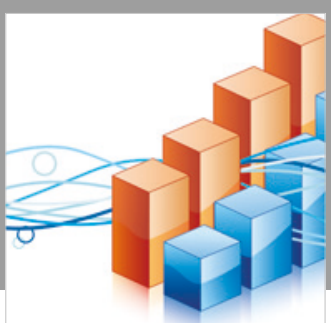

Advances in

Operations Research

\section{-n-m}
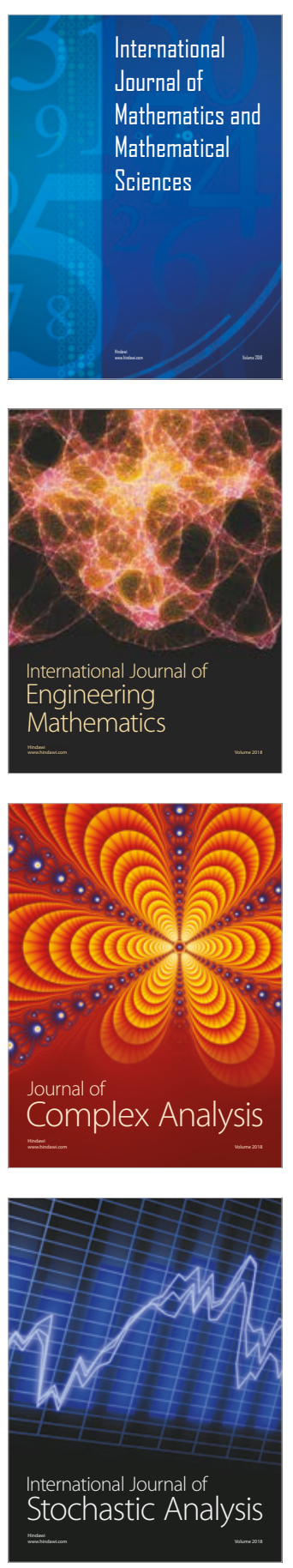
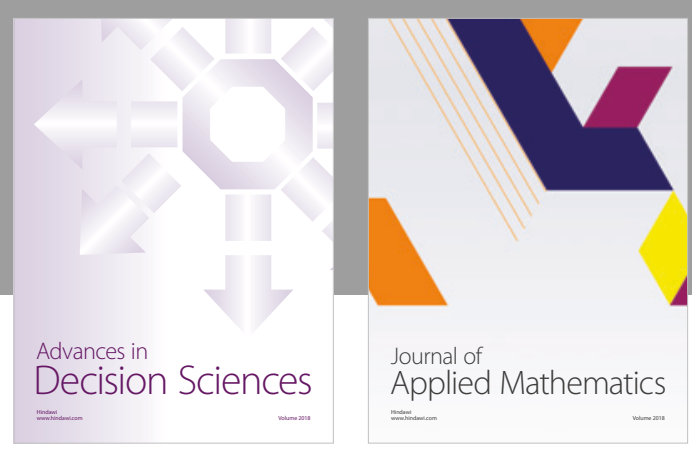

Journal of

Applied Mathematics
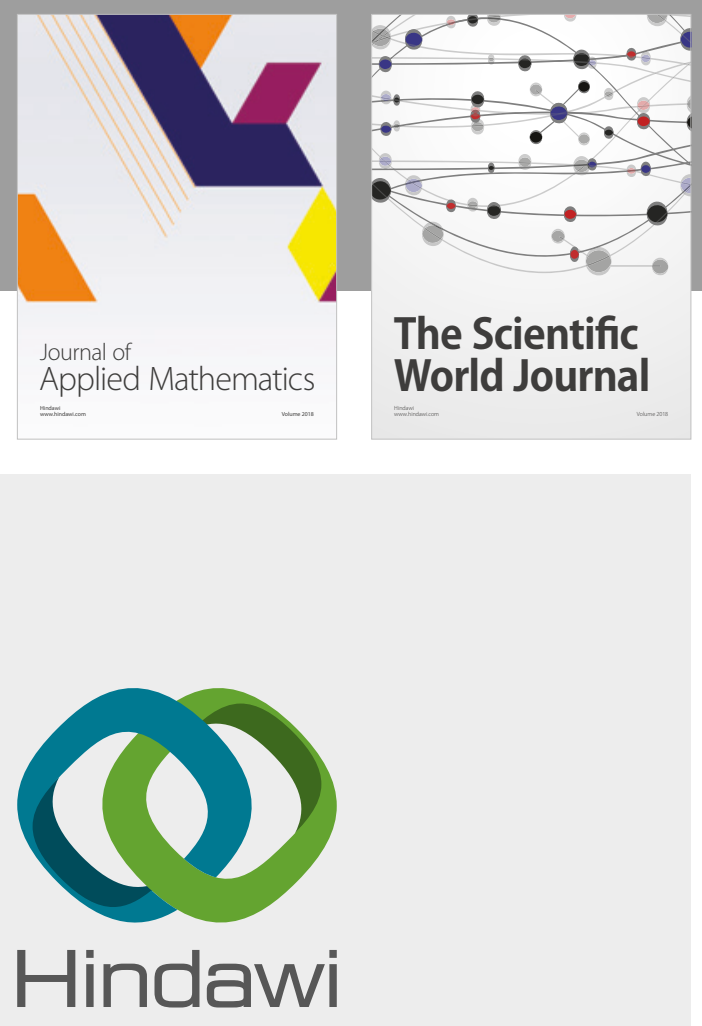

Submit your manuscripts at

www.hindawi.com

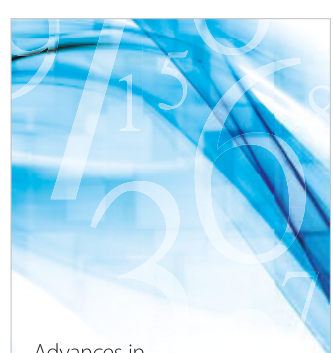

Advances in
Numerical Analysis
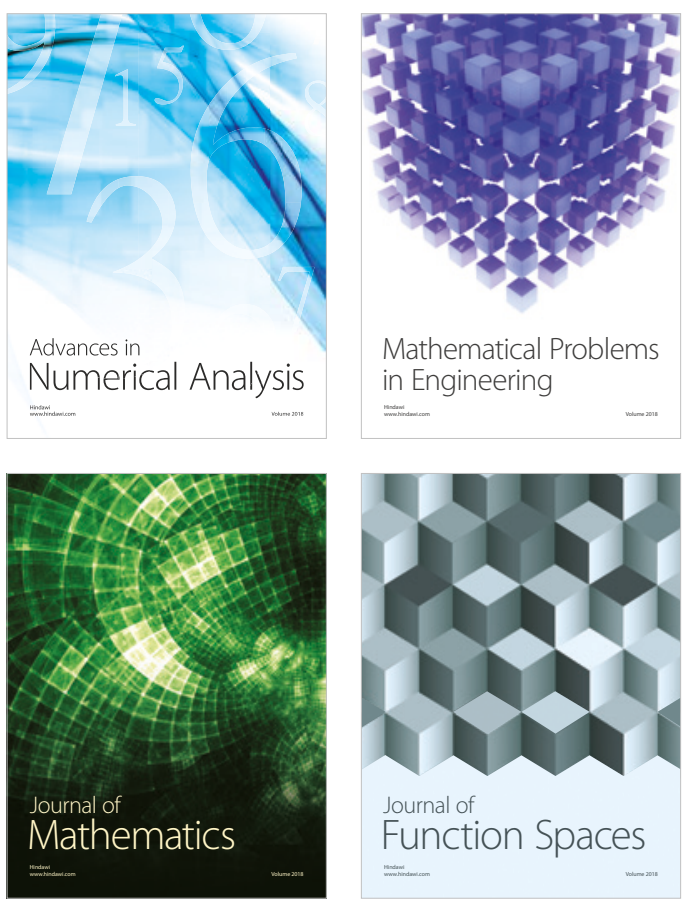

Mathematical Problems in Engineering

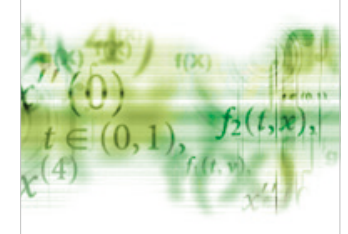

International Journal of

Differential Equations

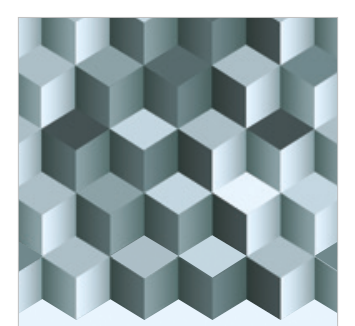

Journal of

Function Spaces

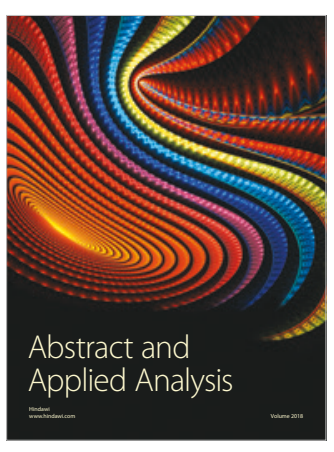

The Scientific

World Journal

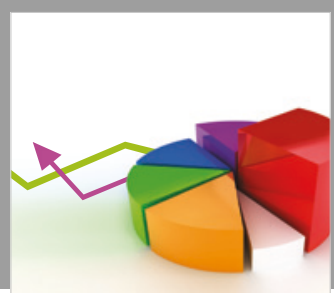

Journal of

Probability and Statistics
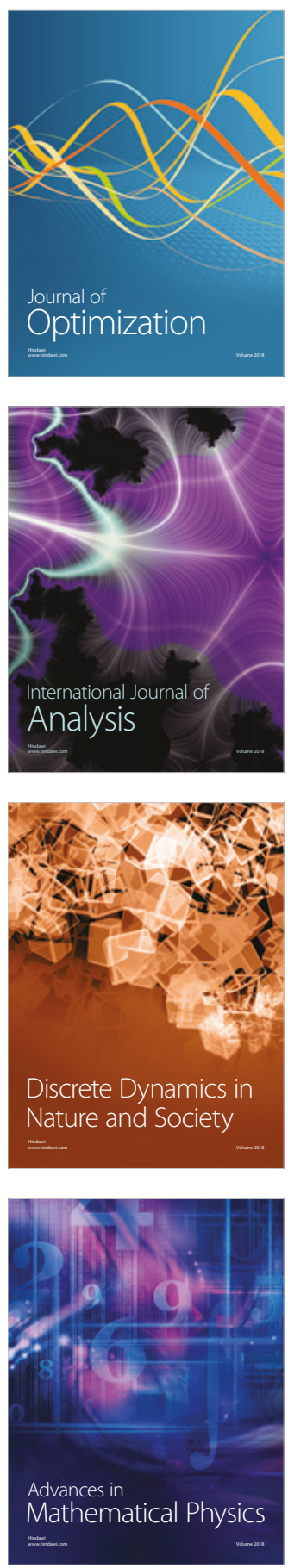